Journal of Advanced Research in Fluid Mechanics and Thermal Sciences

\title{
Numerical Simulation of Fluidic Thrust Vectoring in The Conical Nozzle
}

\author{
Kbab Hakim ${ }^{1,}{ }^{*}$, Hamitouche Toufik ${ }^{1}$, Bergheul Said ${ }^{1}$ \\ Université de Blida1, Aeronautics and Space Studies Institute, aeronautical sciences laboratory, BP270 route de Soumàa, Blida, Algeria
}

\section{ARTICLE INFO $\quad$ ABSTRACT}

\section{Article history:}

Received 20 January 2020

Received in revised form 14 April 2020

Accepted 21 April 2020

Available online 31 July 2020
Keywords:

CD Nozzle; TVC; SVC; NPR; CFD; NPR; SPR

\begin{abstract}
The application of fluid injection into the divergent portion of a supersonic nozzle to release the pushed vector is an attractive alternate to conventional thrust vectoring systems. Indeed, it can replace mechanical complexes. In recent years, this new thrust vectoring technology is the subject of numerous experimental and numerical researches, and has allowed countless applications due to its many advantages. A thrust vectoring concept is currently being applied to new-generation fighter aircraft equipped with jet engine. Fluidic thrust vectoring also finds application in satellite altitude control systems; this principle reduces the number of nozzles used. This study is based on ANSYS-FLUENT and analyzes the different phenomena involved. The results of thrust vectoring performances (angle of deviation, efficiency, lateral forces, etc.) are compared to those in the bibliography.
\end{abstract}

Copyright @ 2020 PENERBIT AKADEMIA BARU - All rights reserved

\section{Introduction}

In the atmospheric layers where the use of the aerodynamic response of the system to control of the vehicle proves to be ineffective, the control using the thrust becomes a necessity. In these conditions, several systems can be envisaged for thrust control. Conventional methods are generally based on the use of mechanical or electromechanical actuators for thrust orientation, such as hydraulic cylinders, or retractable flaps in the case of fighter planes for pivoting the engine nacelle. An interesting alternative to mechanical thrust control systems is possible by fluidic control of the flow inside the nozzle. Indeed, it provides simplicity of use, a substantial gain in weight, a better dynamic response and smaller loss in the thrust specific impulse. Several studies of fluid injection to deflect the thrust have been undertaken around the world. By contrast, experimental studies are mainly conducted in the United States (NASA) and France (ONERA). All this research and studies have been carried out to study and understand the impact of thrust vectoring on the performance of axisymmetric nozzles. In 1977, Masuya et al., [1] performed experimental tests of a $2.4 \%$ injection by a circular orifice of $4 \mathrm{~mm}$ diameter in an axisymmetric nozzle. The study focused mainly on the flow

\footnotetext{
* Corresponding author.

E-mail address: k71.hakim@gmail.com
}

https://doi.org/10.37934/arfmts.73.2.88105 
structure and the distribution of the internal pressure at the level of the injector. Also, during the same year, Wing and Giuliano [2] conducted an experimental study on secondary injection in an axisymmetric nozzle with a $60^{\circ}$ annular slot. This study conducted in the Langley center (NASA), showed that an axisymmetric nozzle can be vectorized in the same proportions as a plane nozzle. Wing and Giuliano [2] obtained a maximum vector angle of $18^{\circ}$ for a SPR $=1.5$ (injection rate of $12 \%$ ), with a deviation of $1.5 \%$ of the injected flow rate. In 1997, Abeyounis and Bennet Jr. [3] studied the influence of the position of the injection on the deflection angle and showed that this angle increases with the total pressure ratio SPR up to a maximum value to then decrease, because of the impact of the shock on the opposite wall of the injector. In 1998, Flamm [4] developed an experiment based on a counter flow model that was tested on a dual flow propulsion system. This concept has shown many advantages in addition to a reduction in weight, a reduction of noise is observed. The tests were performed under static conditions with an NPR ranging from 3.5 to 10 and secondary suction pressures $\Delta \mathrm{P}_{\text {slot }}=0.5$ to $0.78 \mathrm{psi}$. The design condition of the primary nozzle is NPR $=7.82$. However, the potential disadvantage of the counter flow method is the attachment of the primary jet to the suction collar under certain conditions and geometrical configurations. The problem is not easily controllable, but the method has enormous promise if the attachment of the jet can be completely avoided through the design of an adapted nozzle. For example, the results obtained for a vector angle and thrust coefficient for an NPR $=8$ are respectively $12^{\circ}$ and 0.945 with a secondary flow less than $1 \%$ of the primary weight rate. A maximum thrust vector angle of $15^{\circ}$ is obtained at an NPR $=5$ and the thrust coefficient becomes 0.92 . The main objective of the numerical study by Hunter and Deere [5] was to understand the physics of counter-flow shear layers and to make the first CFD simulations of thrust vectoring using 2D two-dimensional computation. The design condition of the primary nozzle is $N P R_{d}=7.82$. Simulation was done for NPR $=8$ and slit pressures between 1 and 6 psi at ambient temperature. The suction parameter $\lambda$ is defined as follows:

$\lambda=\frac{\left(P_{3}-P_{2}\right) L}{\left(\rho_{1} U_{1}^{2}\right) H}$

This variable is used to represent experimental and numerical data. For the same configuration, the jet attachment occurs respectively in simulation and experimental for values of $\lambda$ greater than 0.4 and 0.22 . The very small difference between these results shows the difficulty that is encountered for the jet control. On the other hand, a good correlation between the experimental and numerical computation is observed for the vector angle for $\lambda=0.22$. 2D simulations that do not include viscous side walls effects predict thrust efficiency with a high rate of between $0.5 \%-0.7 \%$ compared to experimental data. The simulation also revealed that in addition to the thrust generated, a counter flow shear layer was developed at the suction zone, which proves that counter flow thrust vectoring does not depend on primary and secondary flows but, on the control of the asymmetrical separation that is modified by the applied suction. In 2003, an experimental and numerical study in a convergent divergent nozzle plane CD-2D was conducted by Waithe and Deere [6], these works have highlighted the effects of the number of injections on the thrust vectoring (one or two injectors). For an injector (at an NPR $=4.6$ and a SPR $=0.7$ ), the results obtained show a strong influence of the ambient pressure prevailing downstream of the injector and the thrust vector angle obtained is around $7^{\circ}$. In 2014, Deng et al., [7] proposed an analytical model to study the depth penetration and the pressure distribution for the injector. The results obtained were adequate with experimental data. Spaid and Zukoski [8] proposed a mathematical model based on the calculation of the effective height of the obstacle equivalent to the injected jet. Shi et al., [9] studied the jet attachment and control method numerically, and conclude that a hysteresis phenomenon existed in the control course. In 2014, Zhaomiao et al., [10] demonstrated that the $M_{a}$ number has a great influence on the performance vector. 
Later, Mnafeg et al., [11] investigated the fluidic thrust vectoring by SVC using orifice injector. Hunter [12] concluded that for higher NPR (NPR $\geq 2)$, the separation is not the result of a stronger shock/boundary layer interaction, but it comes through the natural tendency of an over-expanded nozzle flow. Other studies have shown that the best performance of thrust vectoring was obtained when the injection position is moved afterward [13-15]. They concluded that, at a low pressure ratio, an SVC nozzle with a two injection slots was more efficient compared to SVC nozzle with a single slot, also it was noted that, the angle vector increases by $50 \%$ with the reduction of the injection angle. In 2018, Jingwei et al., [16] studied the flow characteristics and vector performances, in 2D and axisymmetric SVC nozzle, were investigated numerically. The affecting factors including nozzle pressure (NPR), secondary pressure ration (SPR) and free stream $M_{a}$ numbers were considered. Ferlauto and Marsilio [17] studied the numerically the dynamic response of the dual throat nozzle in the open and closed-loop control. The effect of chemical reactions on fluidic thrust vectoring of an axisymmetric nozzle was studied by Chouicha et al., [18].

\section{Analysis of Fluidic Thrust Vectoring}

\subsection{Parameters of Fluid Thrust Vectoring}

To improve the performance of the fluidic thrust vectoring, it is necessary to know the properties of the transverse injection. SVC (shock control vector) is one of the approaches that use the shock wave to control the thrust direction. The flow region of the rocket nozzle would be divided into main supersonic flow and secondary injection flow as shown in Figure 1. The vector angle which represents the deflection angle between the longitudinal axis of the nozzle and the thrust vector that is given,

$\delta=\arctan \frac{F_{y}}{F_{x}}$

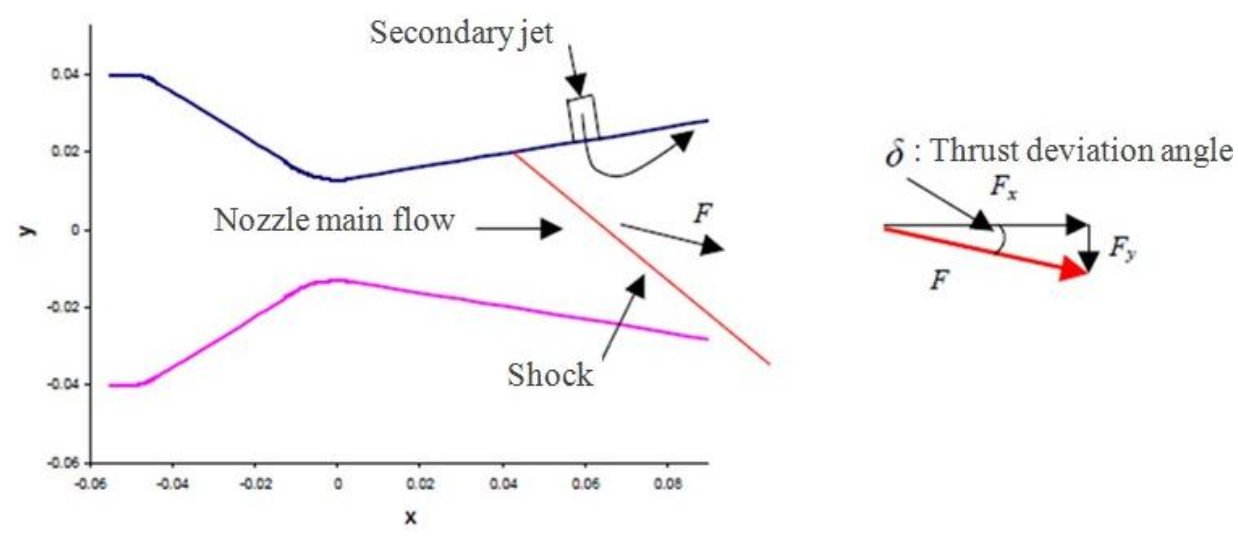

Fig. 1. Simplified scheme of SITVC in axisymmetric C-D nozzle

The thrust vectoring efficiency is defined by the ratio between the deflection angle and the injection rate,

$\eta=\frac{\delta}{100 * \frac{m_{j}}{m_{i}}}$

Amplification factor which makes it possible to compare the different efforts involved

$$
K=\frac{F_{y}}{F_{x j}^{0}}
$$


The ideal thrust is obtained when the flow expands to ambient pressure Pa. After an isentropic expansion, its speed is given by

$V_{i}=\left[\frac{2 \gamma r T_{i 0}}{\gamma-1}\left(1-\left(\frac{P_{a}}{P_{i 0}}\right)^{\gamma-1 / \gamma}\right)\right]^{1 / 2}$

This allows calculating the thrust by the equation below

$F_{\text {idéal }}=m_{l} V_{l}=m_{l}\left[\frac{2 \gamma r T_{l 0}}{\gamma-1}\left(1-\left(\frac{P_{a}}{P_{l 0}}\right)^{\gamma-1 / \gamma}\right)\right]^{1 / 2}$

In this case the thrust coefficient is given as follows

$C_{f}=\frac{\sqrt{F_{x}^{2}+F_{y}^{2}}}{F_{\text {idéal }}}$

\subsection{Analytical Modeling}

The analytical model used in this study is inspired by the Spaid and Zukoski approach [8]. This is based on the calculation of the effective height of the obstacle equivalent to the injected jet and the separation distance. The flow field is mainly divided into two parts by the bow shock. The flow field is simplified as shown in Figure 2.

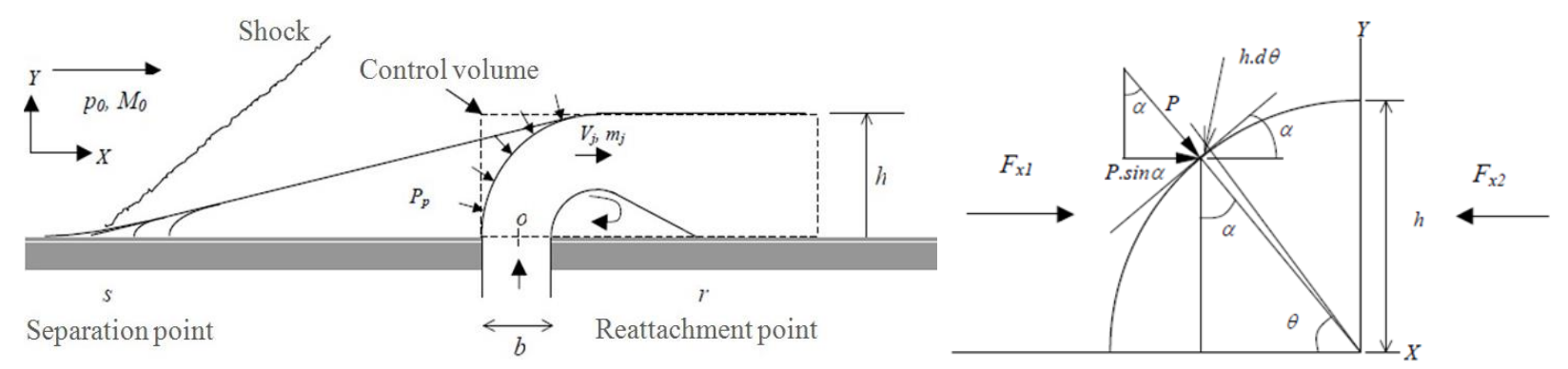

Fig. 2. Sketch of simplified flow field used in the present study

The balance of forces given by the following equation on $\mathrm{x}$

$\sum F_{x}=\frac{d}{d t} \int \rho \cdot v \cdot d y$

where

$\sum F_{x}=F x 1+F x 2$

$F_{x 1}$ is calculated from the following relation,

$F_{x 1}=\int d F_{x 1}=\int P \sin \alpha \cdot d A$

where $C_{p}$ is defined by 
$C_{p}=\frac{P-p_{0}}{q_{0}} \Rightarrow P=p_{0}+C_{p} q_{0}$

$q_{0}$ is the dynamic pressure of the primary flow given by,

$q_{0}=\frac{\gamma_{0} P_{0} M_{0}^{2}}{2}$

The pressure coefficient is written

$C_{p}=2 \sin ^{2} \alpha$

Its maximum value is given by,

$C_{P \max }=2 \sin ^{2} \alpha_{\max }$

Dividing relations 13 and 14 ,

$\frac{C_{p}}{C_{P \max }}=\frac{\sin ^{2} \alpha}{\sin ^{2} \alpha_{\max }}$

$\alpha \max =\pi / 2$ and $C_{p}$ becomes

$C_{p}=C_{P \max } \sin ^{2} \alpha$

In that case, Eq. (10) can be written as follows

$F_{x 1}=\int\left(P_{0} \sin \alpha+q_{0} C_{p \max } \sin ^{3} \alpha\right) d A$

Knowing that: $\quad d A=h . d \theta$ et $\sin \alpha=\cos \theta \quad$ we'll have

$F_{x 1}=\int_{0}^{\pi / 2}\left(P_{0} \cos \theta+q_{0} C_{p \max } \cos \theta^{3}\right) h .1 . d \theta$

Finally,

$F_{x 1}=\left(P_{0}+\frac{2}{3} q_{0} C_{P \max }\right) h$

To calculate $F_{x 2}$, we assume that the downstream pressure of the control volume $p_{a v}$ then,

$F_{x 2}=P \cdot A=-p_{a v} A=-p_{a v} h .1$

Eq. (11) can be written,

$\sum F_{x}=\left(P_{0}-P_{a v}+\frac{2}{3} q_{0} C_{P \max }\right) h$

The secondary jet is injected perpendicular to the flow, its momentum after deflection is 
$\frac{d}{d t} \int \rho \cdot v \cdot d y=m_{j} \cdot v_{J}$

The one-dimensional isentropic expansion leads to the following relation for the speed $V_{j}$

$V_{i}=\left[\frac{2 \gamma r T_{i 0}}{\gamma-1}\left(1-\left(\frac{P_{a}}{P_{i 0}}\right)^{\gamma-1 / \gamma}\right)\right]^{1 / 2}$

where $m_{j}=m_{c}$ is defined as follows

$m_{\mathrm{J}}=m_{c}=\rho_{c} v_{c} A_{c}=\rho_{c} v_{c} C_{d} b .1$

The critical properties of the gas are,

$\frac{T_{c}}{T_{0 j}}=\frac{2}{\gamma_{j}+1} \quad \frac{P_{c}}{P_{0 j}}=\left(\frac{2}{\gamma_{j}+1}\right)^{\gamma / \gamma-1} \quad \frac{\rho_{c}}{\rho_{0 j}}=\left(\frac{2}{\gamma_{j}+1}\right)^{1 / \gamma-1}$

From which we obtain the equation of the momentum of the injected fluid

$m_{j} v_{j}=C_{d} b 2 \gamma_{j} P_{0 j}\left(\frac{2}{\gamma_{j}+1}\right)^{\frac{1}{\gamma_{j}-1}}\left[\frac{1}{\gamma_{j}+1} \frac{1}{\gamma_{j}-1}\left(1-\left(\frac{P_{j}}{P_{0 j}}\right)^{\frac{\gamma_{j}}{\gamma_{j}-1}}\right)\right]^{1 / 2}$

By substituting Eq. (25) and Eq. (21) in Eq. (8) we obtain

$h=\frac{2 c_{d} b P_{0 j} \gamma_{j}}{P_{0}-P_{a v}+\frac{1}{3} P_{0} \gamma_{0} M_{0}^{2} C_{P \max }}\left(\frac{2}{\gamma_{j}+1}\right)^{\frac{1}{\gamma_{j}-1}}\left[\frac{1}{\gamma_{j}^{2}-1}\left(1-\left(\frac{P_{j}}{P_{0 j}}\right)^{\frac{\gamma_{j}}{\gamma_{j}-1}}\right)\right] 1 / 2$

The above equation provides an evaluation of the equivalent step height which depends on the main parameters of the phenomenon and takes into account the expansion effect in the nozzle. To calculate $\mathrm{C}_{\mathrm{oma}}$ we will use the maximum pressure coefficient.

$C_{P \max }=\frac{P_{01}-p_{0}}{\frac{1}{2} \rho_{0} v_{0}^{2}}$

Using the normal shock theory, we calculate

$\frac{P_{01}}{P_{0}}=\left[\frac{(\gamma+1)^{2} M_{0}^{2}}{4 \gamma M_{0}^{2}-2(\gamma-1)}\right]^{\frac{\gamma}{(\gamma-1)}}\left[\frac{1-\gamma+2 \gamma M_{0}^{2}}{\gamma+1}\right]$

And $\mathrm{C}_{\mathrm{pmax}}$ is defined as follows

$C_{P \max }=\frac{2}{\gamma M_{0}^{2}}\left\{\left[\frac{(\gamma+1)^{2} M_{0}^{2}}{4 \gamma M_{0}^{2}-2(\gamma-1)}\right]^{\frac{\gamma}{(\gamma-1)}}\left[\frac{1-\gamma+2 \gamma M_{0}^{2}}{\gamma+1}\right]-1\right\}$

The presence of the secondary injection through a slit in a nozzle causes an oblique shock and consequently a separation of the boundary layer. Assuming that the line of the primary jet 
downstream of the shock is tangent to the circle of radius $h$ established by the injected jet Figure 3 . This line is characterized by the angle $\varepsilon$, assimilated to the deflection angle, behind an oblique shock wave on the wall of the nozzle. The classic oblique shock equations are used to calculate the flow deflection angle and the separation shock angle respectively.

$\frac{P_{p}}{P_{s(x)}}=\frac{2 \gamma M_{s(x)}^{2} \sin ^{2} \beta-(\gamma-1)}{\gamma+1}$

$\tan \varepsilon=\frac{M_{S(x)}^{2} \sin 2 \beta-2 \cot \beta}{2+M_{s(x)}^{2}(\gamma+\cos 2 \beta)}$

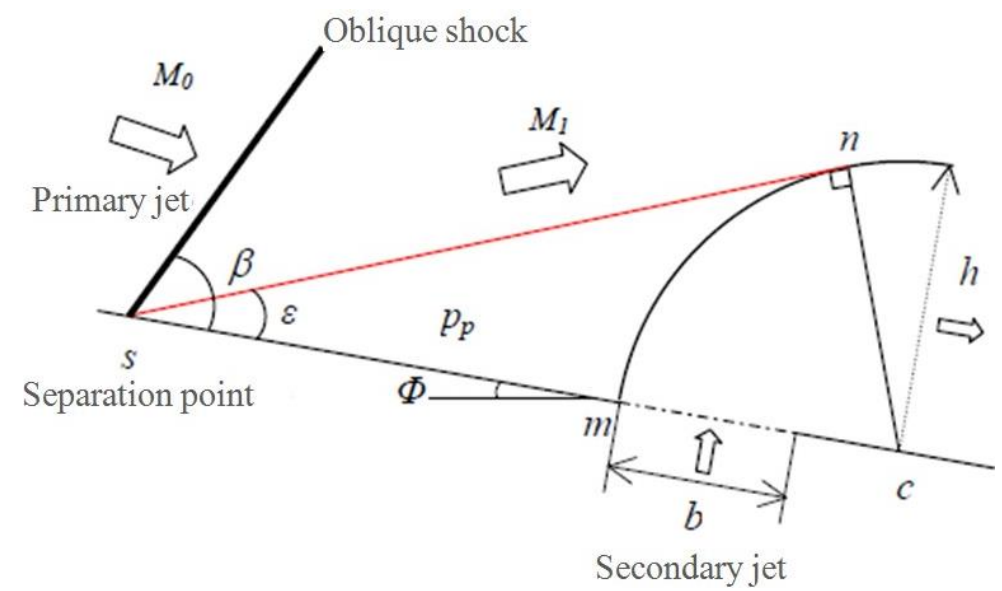

Fig. 3. Separation point calculation method

The abscissa of the separation point $\mathrm{s}$ is obtained by iterative calculation starting from point $\mathrm{m}$ (abscissa from the start of the injector).

In the absence of back pressure at the rear of the nozzle, the secondary jet tends to reattach to the wall. The reattachment jet is characterized by the angle $\psi$ r. The flow passes from state (1) to state (2) after series of detents by modifying its trajectory by an angle $u=\varepsilon$, Figure 4 . Using the Prandtl-Mayer relation, we can easily determine the Mach M2 number of the flow above the step.

$v=\varepsilon=-\sqrt{\frac{\gamma+1}{\gamma-1}} \tan ^{-1} \sqrt{\frac{\gamma+1}{\gamma-1}\left(M_{2}^{2}-1\right)}+\tan ^{-1} \sqrt{M_{2}^{2}-1}$

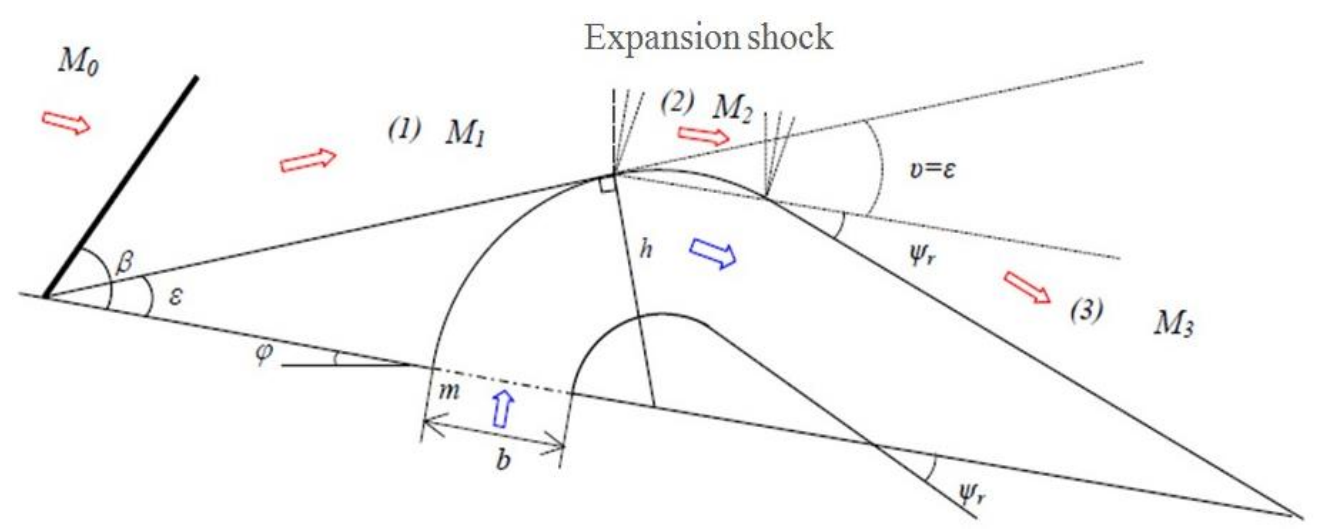

Fig. 4. Representation of the flow above and downstream of the equivalent step 
The flow then passes to state (3) after another series of expansion and a second modification of its trajectory characterized by the angle $\psi \mathrm{r}$. Two main parameters are to be determined in this case. The reattachment angle $\psi r$, this angle is evaluated by a supersonic reattachment criterion, developed by ONERA, as a function of the Mach number M1 of the flow separation and given by the following relation:

$\psi r=32.6-\frac{29.2}{M_{1}}$

The Mach number M3 can be estimated using the Prandtl-Mayer relation as a function of $\psi \mathrm{r}$.

$v=\psi r=-\sqrt{\frac{\gamma+1}{\gamma-1}} \tan ^{-1} \sqrt{\frac{\gamma+1}{\gamma-1}\left(M_{2}^{2}-1\right)}+\tan ^{-1} \sqrt{M_{3}^{2}-1}$

\section{Results and Discussion}

The study was conducted on a convergent divergent nozzle plane CD-2D [12], with a section ratio of 1.8 and a divergence half-angle of $11.01^{\circ}$. The length of the diverging portion $L$ is equal to 0.0577 $\mathrm{m}$. Two types of structured meshes were used during all regions, a mesh A consisting of 182,440 cells and a mesh $B$ composed of 141,600 cells. It has been found that the number of nodes does not really affect the solution. Another $\mathrm{C}$ mesh has been created, with the same number of cells as the mesh $\mathrm{A}$ but more refined at the walls. For the choice of turbulence model, there isn't a general rule to choose such or such model. The choice is related to the nature of the problem: low or high Reynolds number, the behaviour of each model in the near of wall, the ability of each model to predict effectively the separation phenomenon and the desired precision [20]. In our case of study, the k-wSST model is justified in the sense that we want more precision in the near of wall and well the separation region is better captured with the k- $\varepsilon$ standard model. To note, also, that the k- $\omega S S T$ model includes both the advantages of the model $k-\varepsilon$ in regions with high Reynolds number (outside area) and advantages of $k-\omega$ model that performs well in the near of wall (regions with low Reynolds number). The only disadvantage, it requires a more refined mesh that the $k-\varepsilon$ model and it requires $Y^{+}$close to 1 at the wall.

Figure 5 shows the wall pressure distribution for both meshes for NPR=4.6. The results obtained are quite close to each other; however, we opted for mesh A for in order to gain in time. On the other hand, Figure 6 shows the influence of the turbulence model; indeed, two modes were tested (k- $\varepsilon$ and $k-\omega S S t)$, for an NPR $=3$. We noticed that the $k$ - $\omega S S T$ model faithfully reproduces the separation zone. 


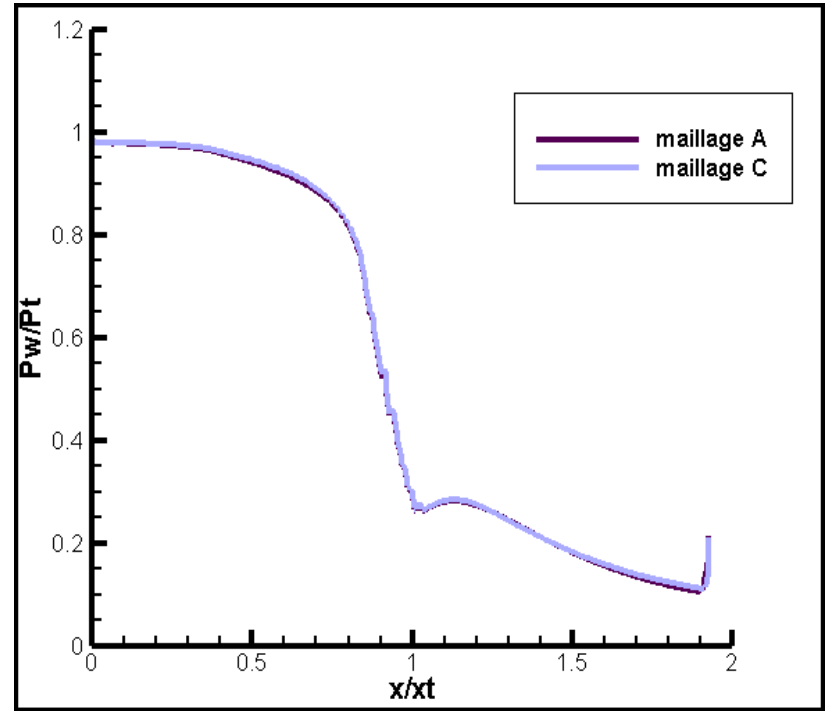

Fig. 5. Distribution of the pressure along the nozzle for different meshes at NPR $=4.6$
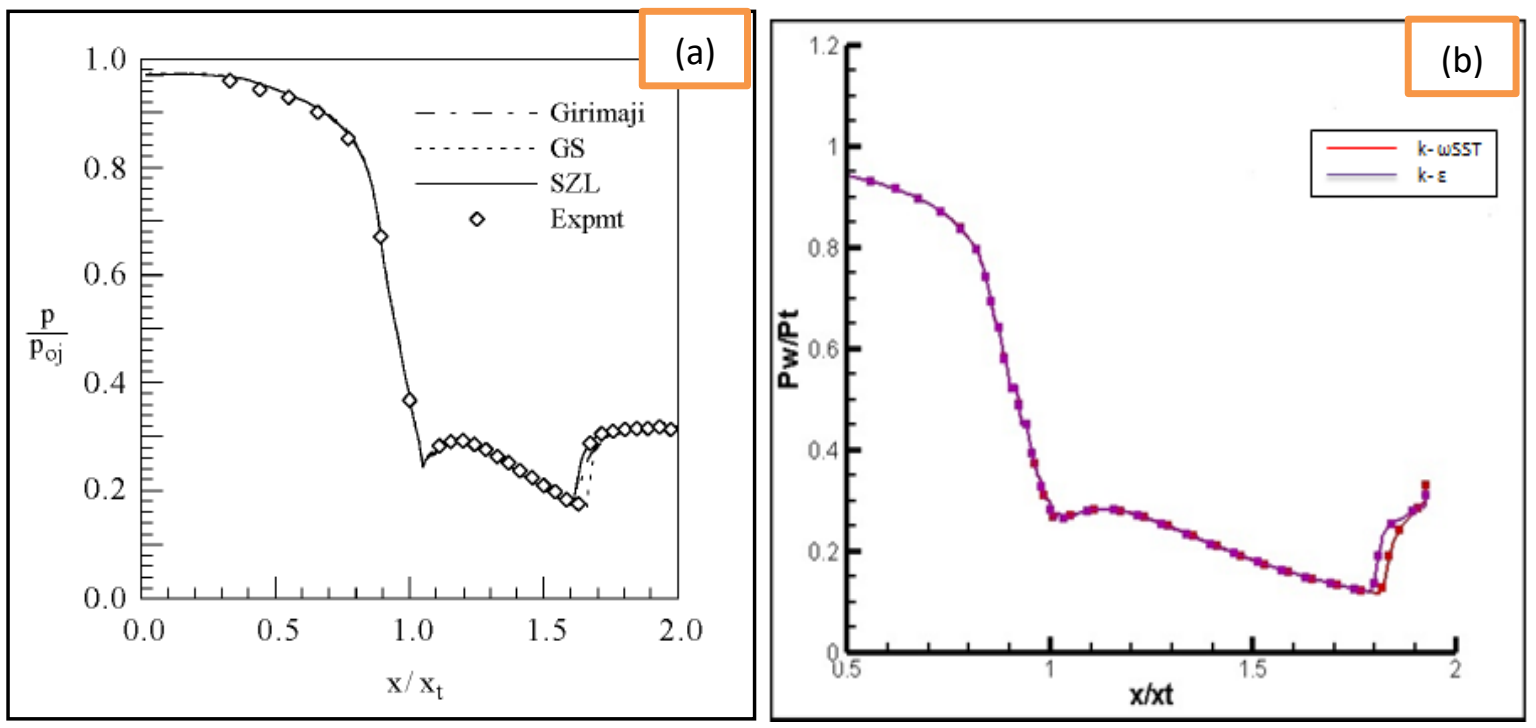

Fig. 6. Influence of the turbulence model NPR = 3, (a): [12]. (b): Our calculations

\subsection{Two-Dimensional Nozzle (2D) Without Secondary Injection}

Figure 7 below shows the distribution of the wall pressure at different NPRs. Note that when the NPR expansion ratio is increased, the point of separation and the position of the right shock move downstream of the nozzle. In addition, we also note that the results of simulations that we obtained are in agreement with those obtained experimentally by Hunter [12]. 

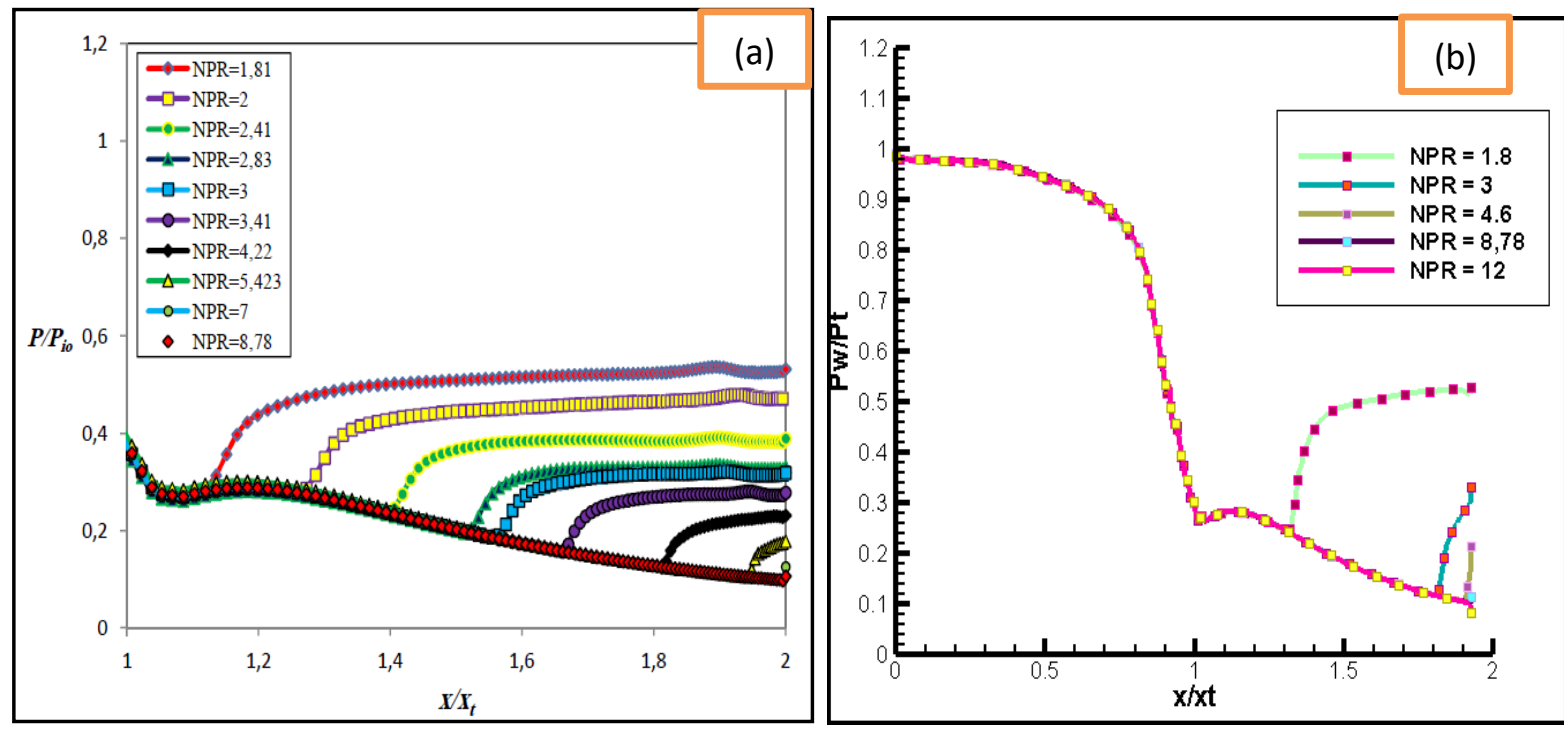

Fig. 7. Distribution of parietal pressure at different NPRs; (a): [12], (b): our numerical calculations

Figure 8 to Figure 10 below represents iso-Mach contours expressed as a function of the ratio of NPR pressures between 1.8 and 12 compared to those obtained by Hunter [12] for NPR (1.8 and 3). The exit Mach number obtained is 1.69 which is close to 1.84 obtained experimentally [12].

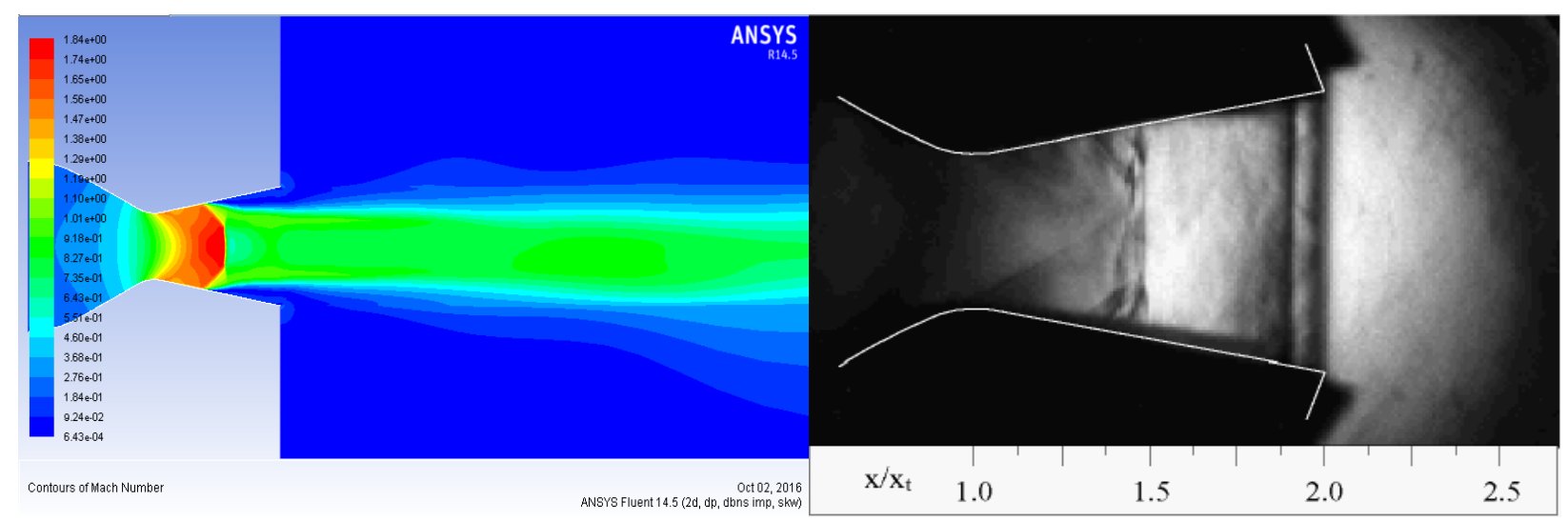

Fig. 8. Experimental stereoscopy [12] and Mach number evolution contour, our calculations for NPR $=1.8$

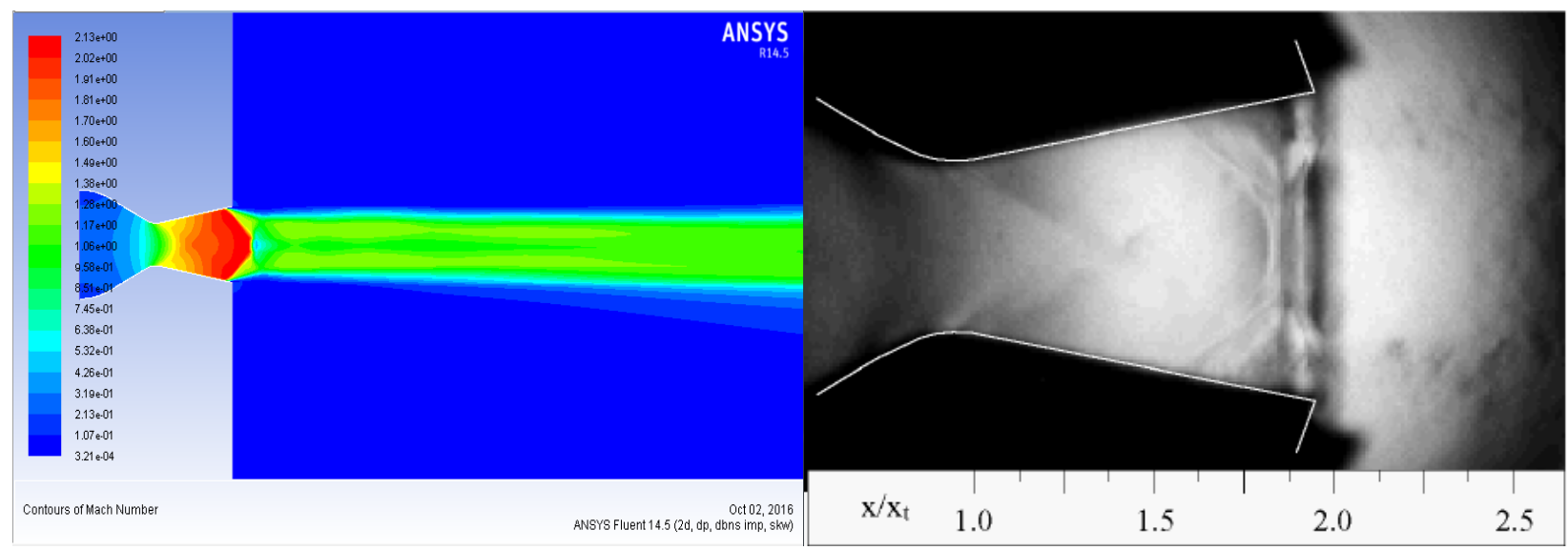

Fig. 9. Experimental stereoscopy [12] and Mach number evolution contour, our calculations for NPR $=3$ 


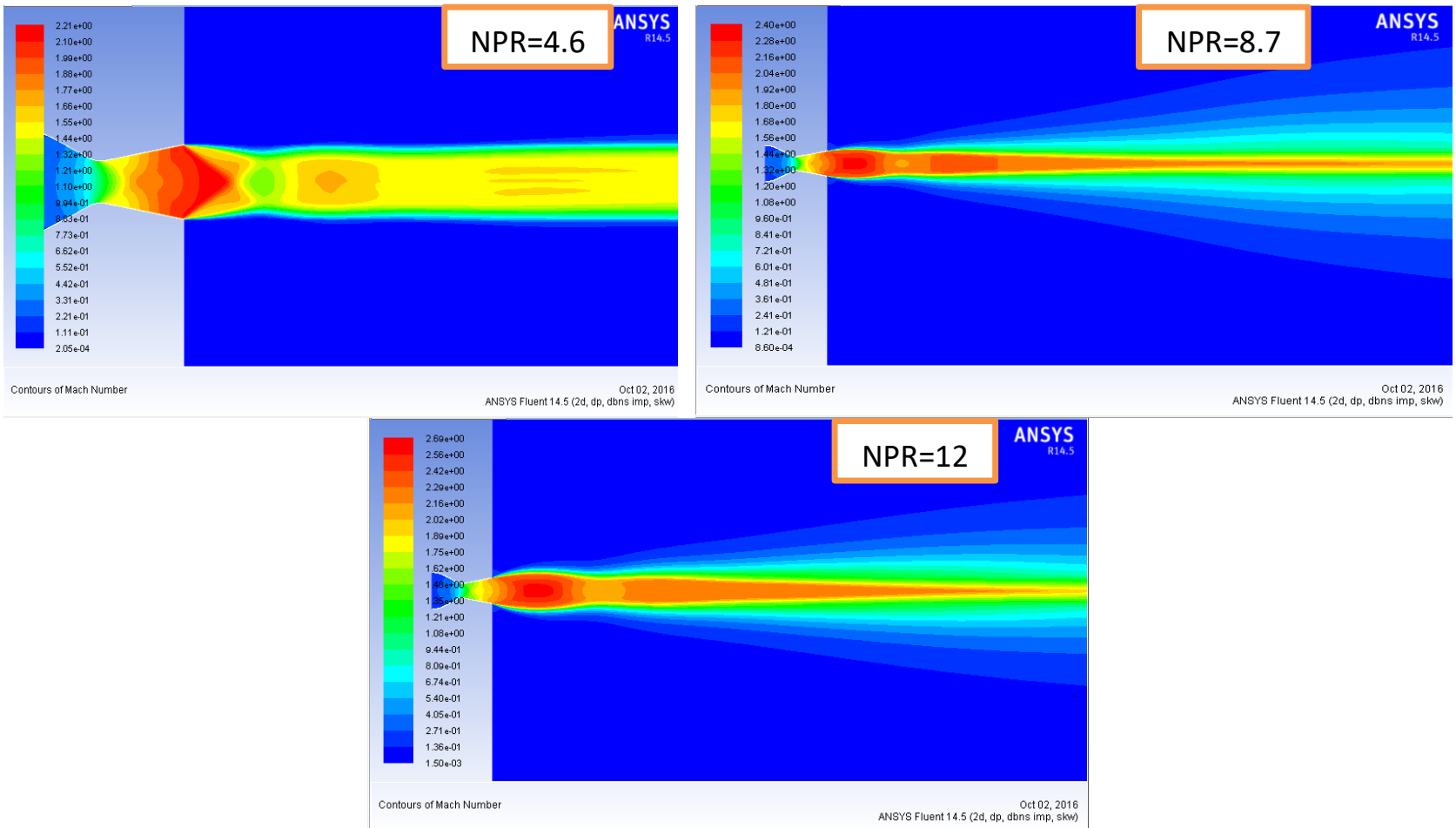

Fig. 10. Mach number evolution for different NPR

For experimental stereoscopy Figure 9 and Figure 10, the shock is channelled the exit of the nozzle, on the other hand for our results the shock is downstream of it. A slight difference was found for both results. By increasing the rate of pressure NPR, the position of Mach's disc moves along the axis of symmetry of the nozzle. The detachment obtained for each pressure ratio is a free detachment. This remark is translated by plateaus pressures which remain constant until the exit of the nozzle. For pressure rates ranging from 1.6 to less than 8.78 Figure 8 to Figure 10 , the ambient pressure is greater than the outlet pressure of the nozzle. In this case, the flow is over-expanded with separation to the value of NPR $=8.78$, where the flow becomes adapted $(\mathrm{Pe}=\mathrm{Pa})$. Above this value, the flow regime is under- expanded, and the outlet pressure is higher than that of the atmosphere.

\subsection{Two-Dimensional Nozzle (2D) with Secondary Injection}

The simulated nozzle is of the convergent-divergent 2D type previously described and owned by NASA. The injection is located at a distance of $0.04 \mathrm{~m}$ from the throat the injection width slot "b" is $0.002 \mathrm{~m}$. Numerical calculations are performed for turbulent and stationary flow. The inlet conditions of the nozzle and of the injection chamber are subsonic with $M=0.1$, while the exit conditions are set at $\mathrm{Pa}=1 \mathrm{bar}$ and $\mathrm{T}=300 \mathrm{k}$. Figure 11 shows the nozzle profile of the nozzle used in 2D calculations. 


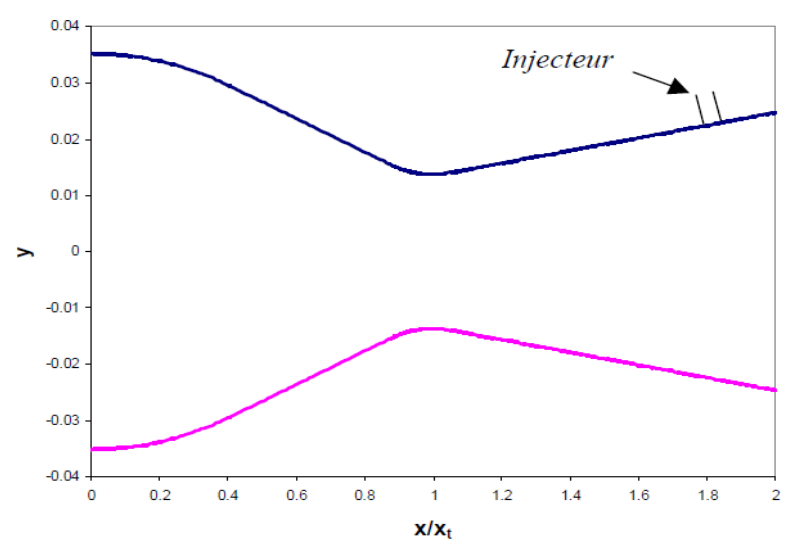

Fig. 11. Nozzle's profile

The simulation is carried out for the case NPR $=4.6$ and SPR $=0.7$ with a turbulence model kWSST. Our numerical calculations are then compared with the experimental work of Waithe and Deere [6]. Figure 12(a) represents experimental stereoscopy compared with that obtained numerically in the form of pressure contours. The presence of an oblique shock upstream of the injection slot is noted on the upper wall. Another shock on the lower wall near the lip of the nozzle is due to the phenomenon of detachment caused by over-expansion. In Figure 12(b), we note the location of the shock which is positioned at $x / x_{t}=1.55$ and $x / x_{t}=1.543$ respectively for the numerical and experimental computations, the relative error is of the order $0.7 \%$ which reinforces our results.

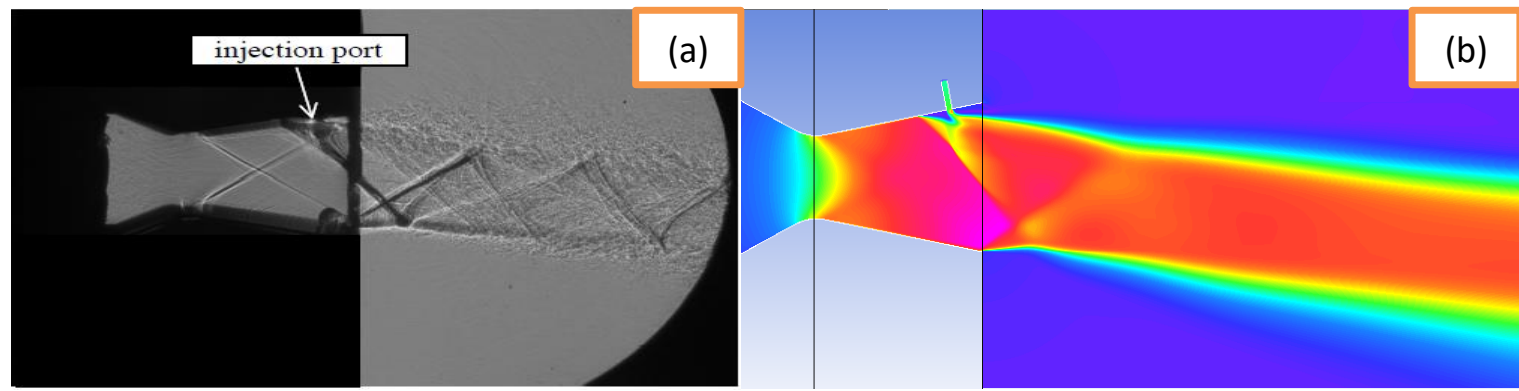

Fig. 12. Pressure evolution, at NPR $=4.6$ and $S P R=0.7,(a):[6]:(b):$ Our simulation

\subsubsection{Variation of SPR total pressure ratio}

Figure 13 shows the parietal pressure evolution for injection rates of $0 ; 2 ; 4$ and $6 \%$ respectively corresponding to SPR=0; $0.4 ; 0.7,1$ and a constant NPR equal to 4.6. The over-expansion regime does not allow the injected jet in the four configurations to reattach to the wall. There is thus an extension of the upstream detachment zone and an increase in the overpressure which prevails, but also the displacement of the shock further upstream by increasing the SPR. A free detachment of the boundary layer was noticed just near the injection slot. 

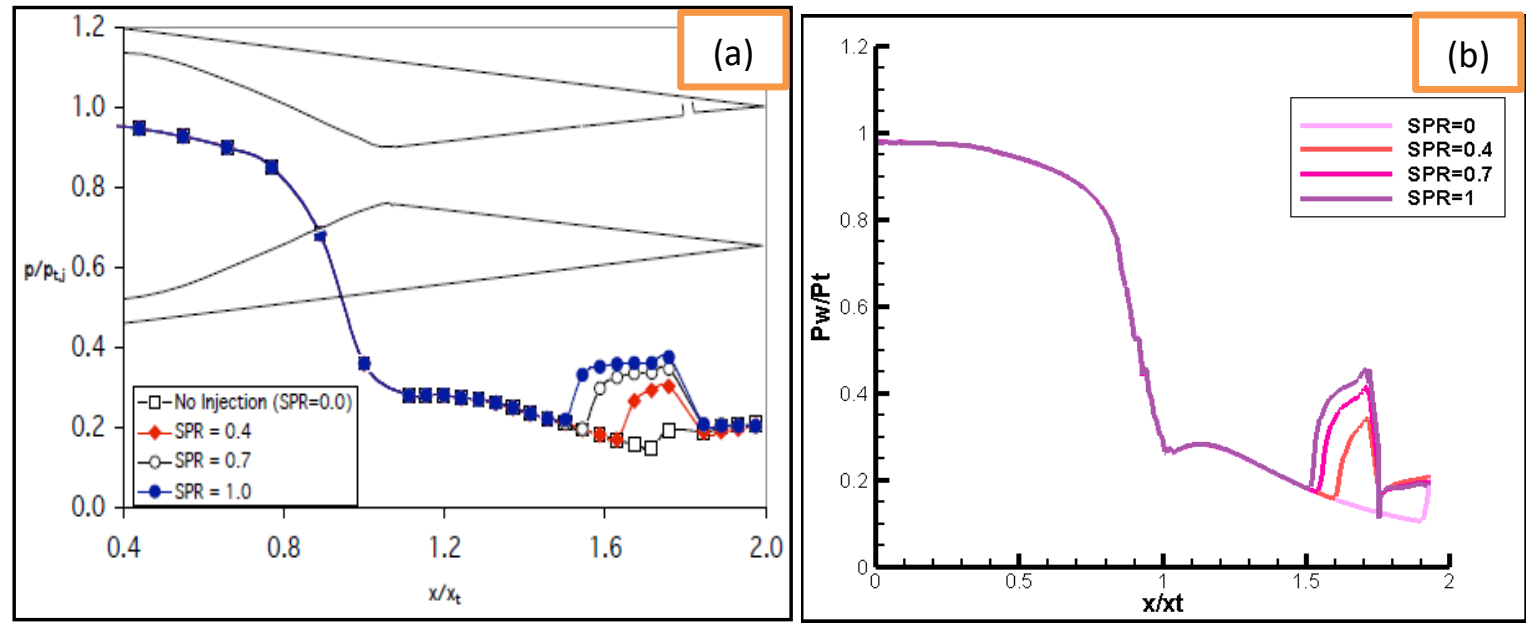

Fig. 13. Wall pressure distribution at NPR $=4.6$ and different NPR (a): [6], (b): our calculations

Figure 14 shows that the flow regime at the inlet of the injector of the fluid injected into the divergent nozzle is sonic $(M=1)$ for an $S P R=1$. This value makes it possible to say that this input is assimilated to a sonic throat of a nozzle.

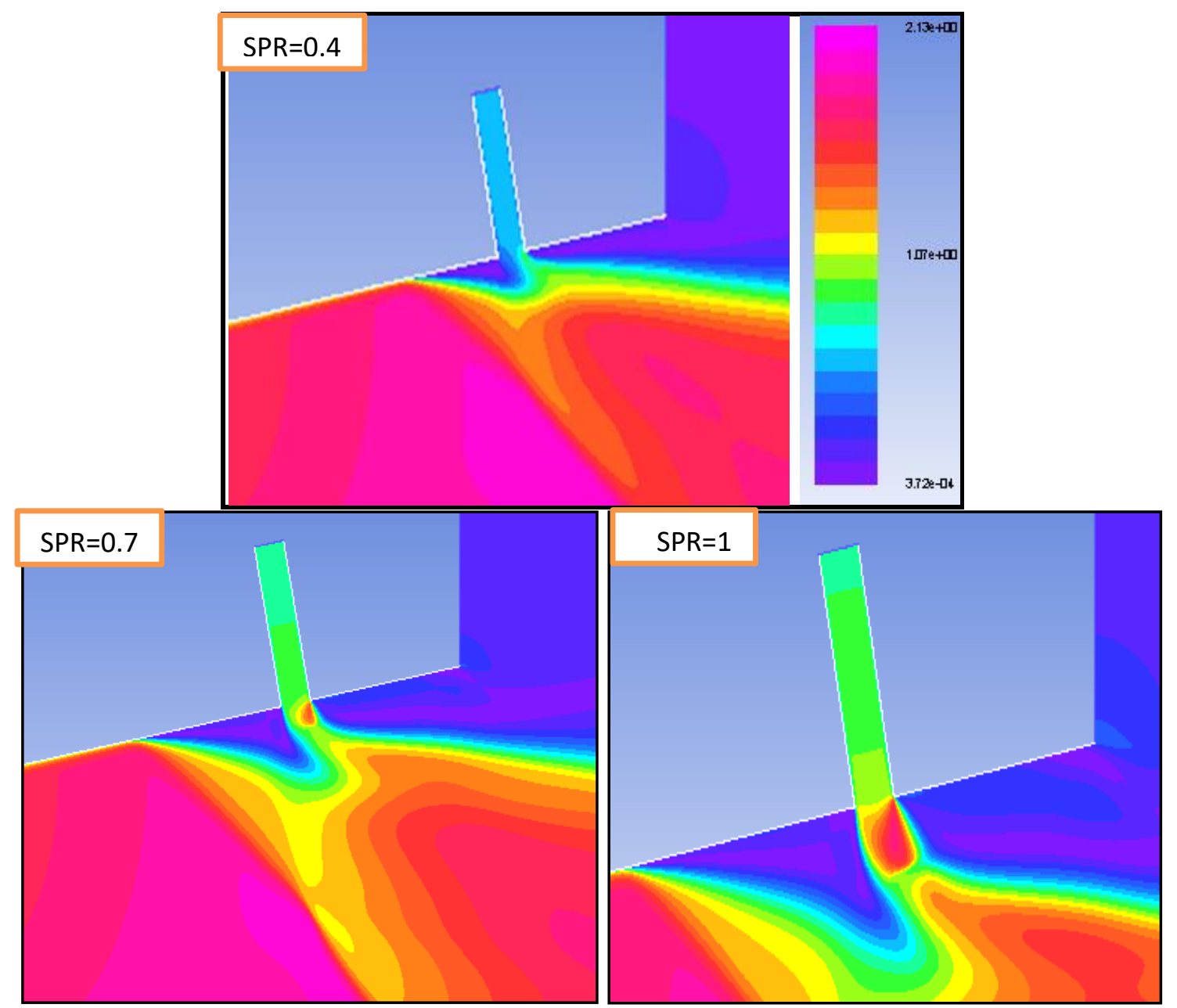

Fig. 14. Mach numbers for different SPRs for NPR $=4.6$ 
Figure 15 gives us the vector angle evolution as a function of SPR. It can be seen that the growth of the angle is proportional to the variation of the SPR, its maximum value is $10.7^{\circ}$ and it is obtained for a SPR equal to 1.

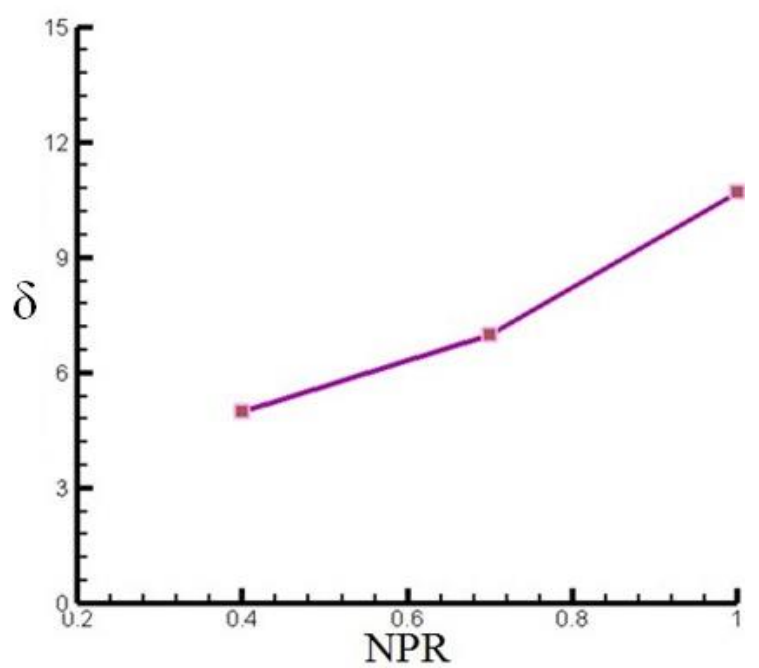

Fig. 15. Vector angle for NPR $=4$

Table 1 shows, for different SPR values, the comparison between the thrust vectoring performances obtained by our calculations and those of the experimental one [6]; we notice that the results are quite close and the relative error is less than $0.5 \%$. Figure 16 represents the wall pressure evolution as a function of the ratio of pressures at NPR $=7$ to SPR $=0.7$. The pressure along the upper and lower walls correlated well with experimental data. The shock is positioned at $x / x_{t}=1.57$ on the upper side which is close to 1.59 obtained experimentally. This slight difference is due to the difference in the processes followed to obtain these results.

Table 1

Summary of our results and experimental results for NPR $=4.6$ and different SPRs

\begin{tabular}{lllll}
\hline & & Our results & Results [6] & Errors \\
\hline & $\mathrm{x} / \mathrm{xt}_{\mathrm{t}}$ & 1.6 & 1.63 & $0.018 \%$ \\
$\mathrm{SPR}=0.4$ & $\delta$ & $5^{\circ}$ & $4.5^{\circ}$ & $0.11 \%$ \\
& $\mathrm{x} / \mathrm{xt}_{\mathrm{t}}$ & 1.57 & 1.55 & $0.012 \%$ \\
$\mathrm{SPR}=0.7$ & $\delta$ & $6.5^{\circ}$ & $7^{\circ}$ & $0.071 \%$ \\
& $\mathrm{x} / \mathrm{xt}_{\mathrm{t}}$ & 1.5 & 1.56 & $0.038 \%$ \\
$\mathrm{SPR}=1$ & $\delta$ & $10.7^{\circ}$ & $9.4^{\circ}$ & $0.013 \%$ \\
\hline
\end{tabular}



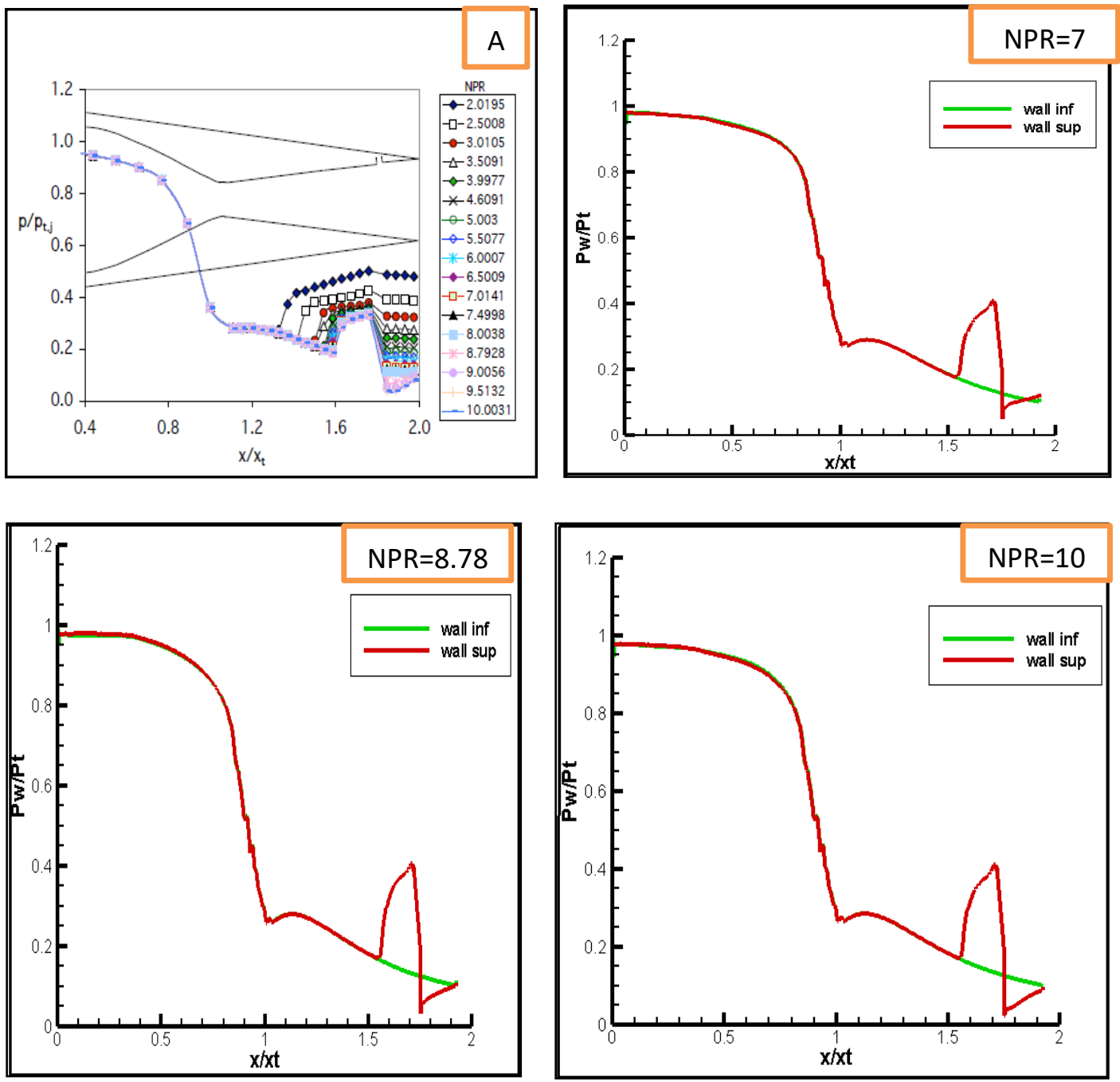

Fig. 16. Wall pressure distribution for different NPR and SPR $=0.7$ (a): [6]

It is noted that the adapted and under expanded flow is obtained respectively for NPR equal to 7.78 and 10.

In the Figure 17 above, we note a jet reattachment increasingly accentuated to the wall when we increase the NPR. When the expansion rate increases the main jet pushes the secondary jet up, this leads to decrease its height $h$. When the NPR is low, the secondary jet penetrates deeper the middle of the main jet is led to a higher height $h$.

The maximum deviation value of the main jet is recorded at NPR $=4.6$, which corresponds to a sharp deviation of the flow in the direction of the injected jet. As the NPR expansion ratio decreases, the deflection angle gradually increases Figure 18.

Table 2 shows the thrust vectoring performances for different NPRs, with an SPR $=0.7$ compared to those obtained experimentally in reference [6]. The consistency of these results has been noted. 


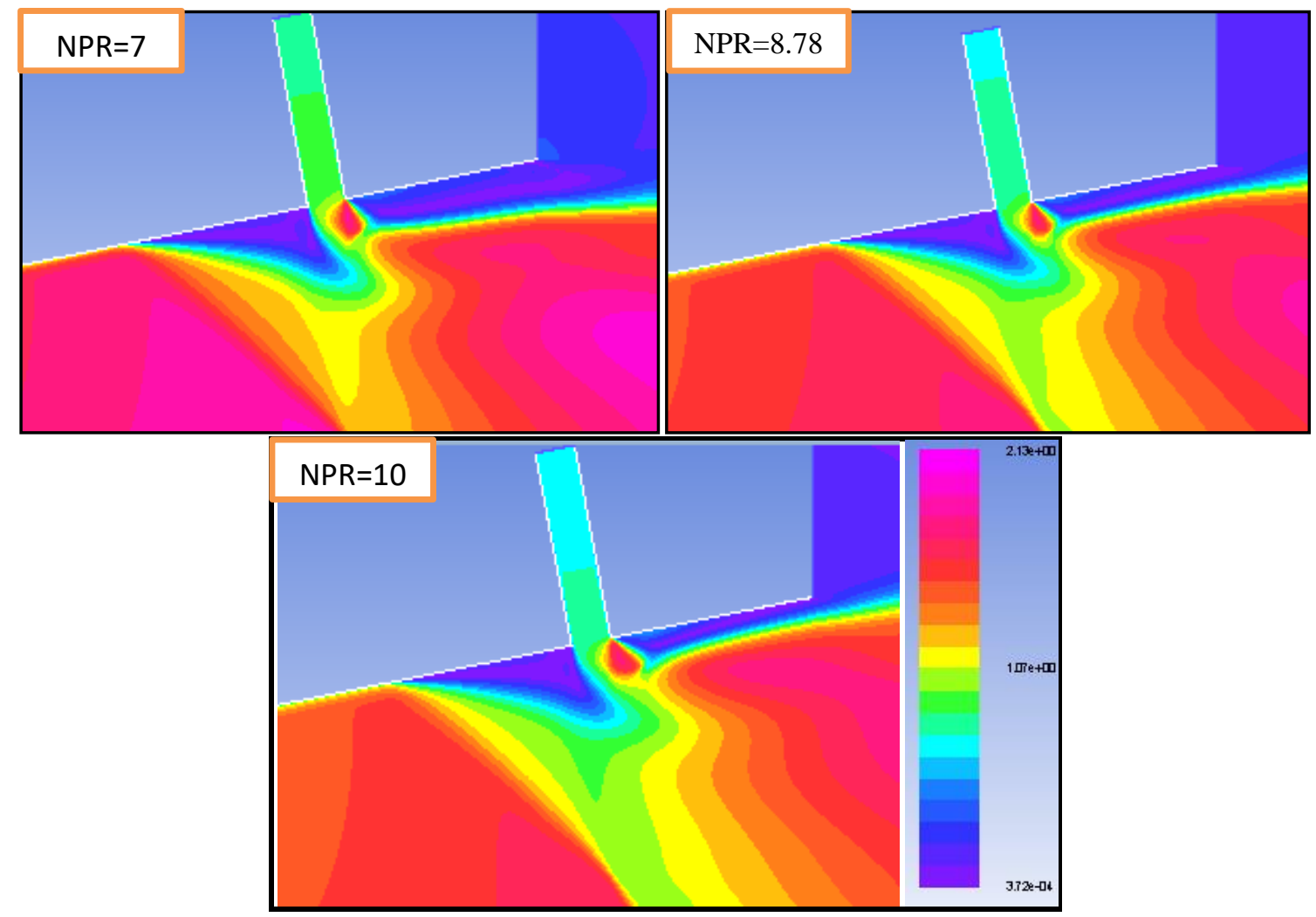

Fig. 17. Mach numbers for different NPRs at SPR $=0.7$

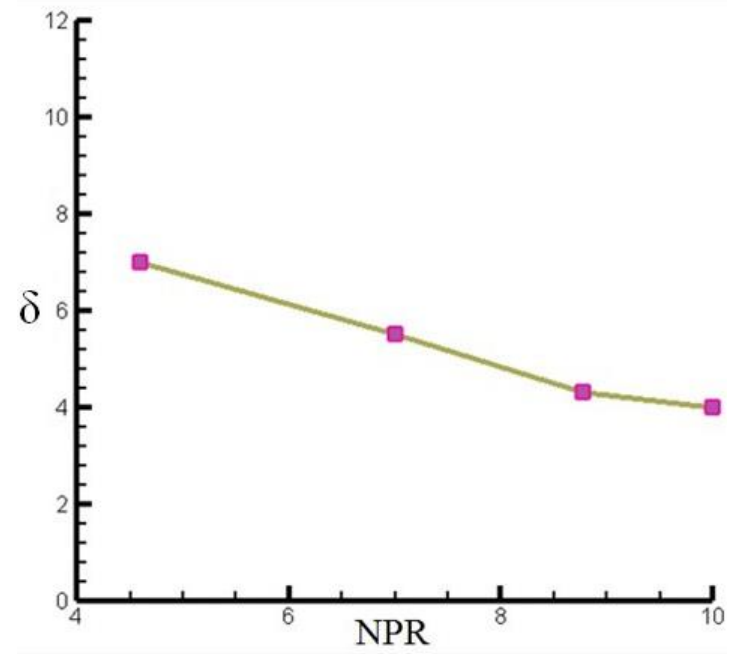

Fig. 18. Vector angle for $S P R=0.7$

Table 2

Summary of our results and experimental results for SPR $=0.7$ and different NPRs

\begin{tabular}{lllll}
\hline & & Our results & Results [6] & Errors \\
\hline & $\mathrm{x} / \mathrm{xt}_{\mathrm{t}}$ & 1.5 & 1.56 & $0.038 \%$ \\
$\mathrm{NPR}=4.6$ & $\delta$ & $10.7^{\circ}$ & $9.4^{\circ}$ & $0.013 \%$ \\
& $\mathrm{x} / \mathrm{xt}$ & 1.57 & 1.59 & $0.0125 \%$ \\
$\mathrm{NPR}=7$ & $\delta$ & $5^{\circ}$ & $4.6^{\circ}$ & $0.086 \%$ \\
& $\mathrm{x} / \mathrm{xt}$ & 1.57 & 1.6 & $0.01875 \%$ \\
$\mathrm{NPR}=8.78$ & $\delta$ & $4.2^{\circ}$ & $4^{\circ}$ & $0.05 \%$ \\
& $\mathrm{x} / \mathrm{xt}$ & 1.57 & 1.61 & $0.025 \%$ \\
$\mathrm{NPR}=10$ & $\delta$ & $4^{\circ}$ & $3.8^{\circ}$ & $0.052 \%$ \\
\hline
\end{tabular}




\section{Conclusion}

The main aim of this work is to make a numerical analysis of the fluidic thrust vectoring by secondary injection in the divergent of a conical nozzle, in order to orient the thrust vector of rocket engines and fighter planes. This principle consists of modifying the pressure field in the divergent so as to produce asymmetrical lateral forces affecting the general deflection of the thrust. The numerical simulations, presented in this study, were carried out with the Ansys-Fluent commercial code based on the resolution of the Navier-Stocks equations of a turbulent and viscous compressible flow, using the k- $\omega S S T$ model as turbulence model. The shock wave interactions in a two-dimensional nozzle were treated in two steps: The first step is done by a calculation based on the variation of the relaxation rates for a case without secondary injection. The second step is performed by the presence of a single injection located at a constant distance from the throat in the divergent part. The results of the modelling of the secondary injection in the divergent were compared with the experimental results obtained in the specialized literature $[6,12]$. The efficiency of the secondary injection has been clearly demonstrated. The results show that the angle vectors obtained are quite close to those obtained with the classical (mechanical) method. The regimes of over-expanded at relatively large total pressure ratios are those for which the thrust vectoring efficiency is the most important. For an over-expanded regime characterized by NPR $=4.6$ and SPR $=0.7$, we obtained $2.1 \%$ efficiency.

\section{References}

[1] Masuya, Goro, Nobuo Chinzei, and Shinichi Ishii. "Secondary gas injection into a supersonic conical nozzle." AIAA Journal 15, no. 3 (1977): 301-302. https://doi.org/10.2514/3.63234

[2] Wing, David J., and Victor J. Giuliano. "Fluidic thrust vectoring of an axisymmetric exhaust nozzle at static conditions." ASME Fluids Engineering Division Summer Meeting (FEDSM97-3228) (1997).

[3] Abeyounis, W. K., and B. D. Bennett Jr. "Static Internal Performance of an Over Expanded Fixed-Geometry, Nonaxisymmetric Nozzle with Fluidic Pitch-Thrust-Vectoring Capability." NASA Paper No. TP-3645 (1997).

[4] Flamm, Jeffrey. "Experimental study of a nozzle using fluidic counterflow for thrust vectoring." In 34th AIAA/ASME/SAE/ASEE Joint Propulsion Conference and Exhibit, p. 3255. 1998. https://doi.org/10.2514/6.1998-3255

[5] Hunter, Craig, and Karen Deere. "Computational investigation of fluidic counterflow thrust vectoring." In 35th Joint Propulsion Conference and Exhibit, p. 2669. 1999.

https://doi.org/10.2514/6.1999-2669

[6] Waithe, Kenrick, and Karen Deere. "An experimental and computational investigation of multiple injection ports in a convergent-divergent nozzle for fluidic thrust vectoring." In 21st AIAA Applied Aerodynamics Conference, p. 3802. 2003. https://doi.org/10.2514/6.2003-3802

[7] Deng, Ruoyu, Fanshi Kong, and Heuy Dong Kim. "Numerical simulation of fluidic thrust vectoring in an axisymmetric supersonic nozzle." Journal of Mechanical Science and Technology 28, no. 12 (2014): 4979-4987. https://doi.org/10.1007/s12206-014-1119-x

[8] Spaid, F. W., and E. E. Zukoski. "A study of the interaction of gaseous jets from transverse slots with supersonic external flows." AIAA Journal 6, no. 2 (1968): 205-212. https://doi.org/10.2514/3.4479

[9] Shi, J-W., Z-X. Wang, X-B. Zhang, and Z-W. Liu. "Study on Counter-Flow Thrust Vectoring Nozzle Jet Attachment and Control." Acta Aerodynamica Sinica 31, no. 6 (2013): 723-726.

[10] Zhao-miao, Liu, Chen Chuan, and F. Shen. "Impact of mach number of external flows on performance of counterflow thrust vectoring nozzle." Journal of Propulsion Technologies 35, no. 3 (2014): 242-248.

[11] Mnafeg, Ibrahim, Azgal Abichou, and Lotfi Beji. "Thrust Vectoring Control of Supersonic Flow Through an Orifice Injector." International Journal of Aerospace and Mechanical Engineering 9, no. 7 (2015): 1352-1358.

[12] Hunter, C. A. "Experimental, theoretical, and computational investigation of separated nozzle flows. The 34th AIAA." ASME/SAE/ASEE Joint Propulsion Conference and Exhibit, NASA Langley Research Center, 1998. AIAA Paper 98-3107, 1998.

https://doi.org/10.2514/6.1998-3107 
[13] Zmijanovic, Vladeta, Viviana Lago, Mohamed Sellam, and Amer Chpoun. "Thrust shock vector control of an axisymmetric conical supersonic nozzle via secondary transverse gas injection." Shock Waves 24, no. 1 (2014): 97 111. https://doi.org/10.1007/s00193-013-0479-y

[14] Shi, Jing-Wei, Zhan-Xue Wang, Zeng-Wen Liu, and Xiao-Bo Zhang. "Effects of secondary injection forms on thrust vector performance of shock vector controlling nozzle." Journal of Aerospace Power 28, no. 12 (2013): 2678-2684.

[15] He, Chengjun, Jianqiang Li, Yaohua Li, and Jinmin Liang. "Influence of secondary injection parameters on performance of shock vector control nozzle." In 21st AIAA International Space Planes and Hypersonics Technologies Conference, p. 2270. 2017. https://doi.org/10.2514/6.2017-2270

[16] Jingwei, Shi, Wang Zhanxue, Zhou Li, and Sun Xiaolin. "Investigation on Flow Characteristics of SVC Nozzles." Journal of Applied Fluid Mechanics 11, no. 2 (2018): 331-342.

[17] Ferlauto, Michele, and Roberto Marsilio. "Open and closed-loop responses of a dual-throat nozzle during fluidic thrust vectoring." In 52nd AIAA/SAE/ASEE Joint Propulsion Conference, p. 4504. 2016. https://doi.org/10.2514/6.2016-4504

[18] Chouicha, Rachid, Mohamed Sellam, and Said Bergheul. "Effect of chemical reactions on the fluidic thrust vectoring of an axisymmetric nozzle." International Journal of Aviation, Aeronautics, and Aerospace 6, no. 5 (2019): 1-24. https://doi.org/10.15394/ijaaa.2019.1377

[19] Khan, Sher Afghan, Abdul Aabid, Fharukh Ahmed Mehaboobali Ghasi, Abdulrahman Abdullah Al-Robaian, and Ali Sulaiman Alsagri. "Analysis of area ratio in a CD nozzle with suddenly expanded duct using CFD method." CFD Letters 11, no. 5 (2019): 61-71. 